\title{
Über die wissenschaftlichen Beziehungen von Hans Ertel und Heinrich Ficker
}

\author{
Von \\ Wilfried Schröder \\ (Vorgelegt in der Sitzung der math.-nat. Klasse am 15. November 2007 \\ durch das w. M. Helmut Moritz)
}

\section{Vorbemerkung}

Im Gegensatz zum englischen Sprachraum befindet sich die Aufarbeitung der Geschichte der geophysikalischen Disziplinen in Deutschland erst im Aufbau. Das ist bedauerlich, denn gerade hier wurden viele Grundlagen der heutigen Meteorologie und Geophysik gelegt. So kann es keinen Zweifel geben, dass gerade auch das Berliner Meteorologische Institut zur Zeit der Wirksamkeit von HEINRICH $[\mathrm{VON}]^{1}$ FICKER sowie HANS ERTEL eine internationale Leitfunktion hatte. Die Wiederkehr des 50. Todesjahres von Akademiemitglied HEINRICH FICKER mag Anlass sein, einige Linien in der Geschichte der Beziehung dieses Wissenschaftlers zu ziehen. Vornehmlich wird man sich dabei auf HANS ERTEL beziehen, dessen Entdeckung FICKER selbst als seine wohl bedeutendste wissenschaftliche Leistung bezeichnete. Von einem Versuch muss indes gesprochen werden, weil viele Unterlagen aus der Zeit der beiden Gelehrten nicht mehr verfügbar sind, so dass auf manche Linie indirekt geschlossen werden muss.

\footnotetext{
${ }^{1}$ Je nach dem verschiedenen Gebrauch der ehemaligen Adelsprädikate in Deutschland und Österreich.
} 


\section{Die Berliner Zeit}

Die meteorologische Forschung hat im Berliner Raum eine lange Tradition. Die Gründung des „Preußischen Meteorologischen Instituts“ im Jahre 1847 bildet dabei in organisatorischer Hinsicht nur den Beginn.

\subsection{Entwicklungslinien}

Auf WilHELM MaHLMANN (1812-1848) folgte HEINRICH WiLHELM Dove (1803-1879), der die meteorologische Arbeit in vielfältiger Weise aktivieren sollte. Eine Neuorientierung des Instituts erfolgte dann durch den Physiker WilHELM VON BEZOLD (1837-1907). In den Folgejahren gewann auch die theoretische orientierte Forschung zunehmend Gestalt, wobei der Name von HERMANN VON HELMHOLTZ (1821-1894) als Beispiel genannt sei. So wurde auch rasch die Bedeutung der Helmholtzschen Wirbelsätze für die Meteorologie gesehen. DOVE und BEZOLD waren es, die die Wirbeldynamik auf die reale Atmosphäre zu übertragen suchten. Erst in unserer Zeit hat indes HANS ERTEL (19041971) ihr jene Form gegeben, die auch für reale Medien anwendbar ist.

Im Jahre 1923 übernahm HEINRICH FICKER als Nachfolger von GUSTAV HELLMANN (1854-1939) das Amt sowie den Lehrstuhl in Berlin. Bereits die Antrittsrede FICKERS vor der Preußischen Akademie lässt einige Aspekte seines Programms, wenn man es so bezeichnen will, erkennen. FICKER war am 28. Juli 1926 zum Ordentlichen Mitglied geworden und hielt am 30. Juni 1927 seine Antrittsrede. Darin hebt er sowohl die Beiträge der österreichischen als auch der skandinavischen Meteorologenschaft hervor. Besonders die klassischen Studien von HANN, EXNER, MARGULES und TRABERT betont er. Interessant ist auch, dass FICKER gerade in den MARGULESschen und EXNERschen Beiträgen wesentliche Vorarbeiten für die später formulierte Polarfronttheorie von BJERKNES sieht. Damit ist etwas angesprochen, das über Jahre hinweg die Gegensätze zwischen der norwegischen Schule einerseits, und der österreichisch-deutschen Schule andererseits, beinhaltet. Dies ist in gewisser Weise fast tragisch zu nennen, denn der Beginn der wissenschaftlichen Beziehungen zwischen BJERKNES und FICKER war überaus freundlich und von aufrichtigem, gegenseitigem Respekt geprägt. Einige Aspekte aus diesen Beziehungen müssen angesprochen werden, weil sie zum Verständnis der späteren Jahre wichtig sind.

\subsection{Bjerknes und Ficker}

Das Problem der „Polarfronttheorie“ kommt einerseits in einem Brief FICKERS an BJERKNES vom 15. Oktober 1920 zum Ausdruck. Darin 
betont er, dass er anlässlich eines Leipziger Besuches mit diesem das Problem erörtern konnte. FICKER beklagt, dass - infolge der Kriegsauswirkungen - seine Arbeiten zu wenig Beachtung gefunden haben. In seiner kritischen Lage bittet er BJERKNES deshalb sowohl um allgemeine Literatur als auch selbst nur solche zur Polarfronttheorie. Unter dem 30. Oktober 1920 schrieb BJERKNES sofort zurïck und bemerkte:

„,.. Leider können wir deshalb nur sehr wenig zurückschicken. Denn seitdem wir den ,,praktischen Weg“ eingeschlagen haben, hat uns die „Praxis“ überwältigt. Im Wesentlichen kann ich Ihnen daher nur ältere Arbeiten schicken, die Ihnen zeigen werden, von welchem abstrakt entlegenen Gebiete ich in die Meteorologie hineingeraten bin. Unwissenheit in der meteorologischen Literatur müssen Sie deshalb auch mir und den Meinigen vergeben. Hier in Bergen sind wir außerdem oder waren wenigstens zu Anfang so ziemlich isoliert.“

Unter dem 12. November 1920 bemerkt FICKER seine Freude über BJERKNES' Hinwendung zur Praxis:

„Dass Sie, hochverehrter Herr Professor, von so abstrakt-theoretischer Seite in die Meteorologie gekommen sind, ist uns ein ungeheuerer Vorteil auch für die meteorologische Praxis. Der Praktiker sieht ja so oft den Wald vor lauter Bäumen nicht.“

Zwei Jahre später macht FICKER in einem Brief an BJERKNES indes bereits auf unterschiedliche Standpunkte aufmerksam, wenn er schreibt:

„Was das Depressionsschema selbst betrifft, so habe ich bezüglich einiger Einzelheiten allerdings eine abweichende Auffassung. Nach den Beobachtungen liegt die Stratosphäre nicht am tiefsten über dem warmen Gebiete, sondern erreicht ihre tiefste Lage erst dann, wenn in den niederen Schichten bereits eine niedrige Kältewelle im Gange ist. Auch bezüglich der Vorgänge an der warmen Front bin ich zum Teil anderer Ansicht. Ich vermisse in Ihrem Depressionsschema z. B. das antizyklonale Entwicklungsstadium mit absteigender Luftbewegung .... . Der Hauptunterschied unserer Auffassungen liegt wohl darin, dass nach meiner Meinung die Vorgänge in der Stratosphäre eine gewisse, aktivere Rolle spielen als ihnen in Ihrer Auffassung eingeräumt wird.“ (FICKER, 27. Januar 1922.)

Das Thema „Rolle der Stratosphäre für das Wettergeschehen“ sollte weiterhin die Beziehungen zwischen BJERKNES und FICKER bestimmen. Es führte auch zu einer gewissen Entfremdung der beiden Schulen. Es wundert nicht, dass zunächst FICKER sowie später ERTEL dieses Thema erneut aufgriffen (siehe ERTEL [3]). Die Unterschiede zwischen BJERKNES und FICKER kommen in einem weiteren Schreiben FICKERS vom 27. Februar 1923 zum Ausdruck:

„... Ich habe über Ersuchen EXNER's für die Meteorologische Zeitschrift ein langes Referat über die Polarfronttheorie geschrieben. Ich erlaube mir, seine 
Korrektur an Sie nach Gastein zu schicken. Dass ich sachlich auf dem Boden der Polarfronttheorie stehe, ist Ihnen ja wohl bekannt. Ich fühle mich aber andererseits verpflichtet, über die ähnlich gerichteten Bestrebungen der österreichischen Meteorologie zu referieren, andererseits habe ich die Empfindung, dass die neuesten Ergebnisse der Theorie-Periodizität der Familien etc. viel mehr als bisher durch empirisches Beweismaterial gestützt werden müssen. Es wäre für mich, obwohl ich ausgesprochener und einseitiger Empiriker bin, natürlich von höchstem Werte, wenn mir Ihr Besuch in Graz Gelegenheit zu einer persönlichen Aussprache mit dem Schöpfer der Polarfronttheorie geben würde."

Der weitere, nur sporadisch vorliegende Briefwechsel betrifft die Vortragsreise von BJERKNES nach Berlin. In einem weiteren Schreiben beglückwünscht FICKER BJERKNES zu dessen Wahl zum Korrespondierenden Mitglied der Berliner Akademie der Wissenschaften (10. Mai 1928).

\subsection{Das Berliner Meteorologische Institut}

Im Jahre 1923 wurde FICKER Professor für Meteorologie an der Universität Berlin sowie Direktor des Preußischen Meteorologischen Instituts. Über seine Absichten schreibt er an BJERKNES noch am 27. Februar 1923:

„Es wäre für mich von umso größerem Werte, als ich in meiner neuen Stellung als Direktor des Preußischen Meteorologischen Institutes meine Hauptaufgabe darin erblicken werde, die wissenschaftliche Tätigkeit des Institutes in modernere, von Ihnen vertretene Bahnen zu lenken.“

In späteren Jahren ergaben sich indes Änderungen in der Organisation des Instituts, das anfangs auch durchaus noch für die Lehrzwecke genutzt wurde. Der größte Teil wurde jedoch 1934 in den „Reichswetterdienst“ “überführt. Das was übrig blieb, bildete das Universitätsinstitut, das FICKER als Direktor leitete (neben seiner Lehrtätigkeit an der Universität sowie an der Akademie der Wissenschaften). Hinzu kommt, dass er von 1928 bis 1945 zugleich Präsident der Internationalen Klimatologischen Kommission war. Vor der erfolgten Trennung hatte die meteorologische Bibliothek Präsenzcharakter, d. h. die vorhandene Literatur konnte an Ort und Stelle eingesehen und genutzt werden. Die Verlagerung zum Reichswetterdienst brachte zwar diesem einen Aufwärtstrend, machte jedoch für das verbliebene Universitäts-Institut neue Überlegungen notwendig. Neben einem Neuaufbau bezüglich der Literatur wurde schließlich eine eigene Schriftenreihe begründet, die FICKER mit ERTEL herausgeben sollte. 
Für die Berliner Zeit war neben FICKER besonders A. DEFANT sowie J. BARTELS wichtig. BARTELS war seit 1927 Privatdozent an der Universität und wurde 1928 Professor. 1934 wurde er Professor für Geophysik an der Forstwirtschaftlichen Hochschule in Eberswalde, 1936 war er Professor für Geophysik an der Berliner Universität und übernahm zugleich die Leitung des Geophysikalischen Instituts in Potsdam. BARTELS war zusammen mit FICKER Referent der ERTELschen Dissertation ,Theorie der durch Variationen des magnetischen Potentials induzierten Erdströme bei ungleichförmiger Leitfähigkeit der Erdrinde “, mit der ERTEL mit Auszeichnung promovierte. Vorübergehend wirkte ERTEL am Meteorologisch-Magnetischen Observatorium in Potsdam, doch konnte es ihn dort nicht lange halten. Zwar hätte es BARTELS sehr gerne gesehen, wenn ERTEL bei ihm geblieben wäre, aber diesen zog es zurück nach Berlin.

Neben der bereits von FICKER beabsichtigten modernen Ausrichtung der Forschung, der auch das spätere Universitäts-Institut galt, wurden auch weitere Teilfragen der Meteorologie umfassend gepflegt. Die Wettervorhersage verblieb dem Institut: FICKER selbst kam täglich zur Mittagsprognose. Arbeiten zum Bio-, Agrar- und zum Lokalklima wurden gefördert. Die theoretische Ausrichtung lag später bei HANS ERTEL, der zudem ab 1938 als apl. Privatdozent die Theoretische Meteorologie vertrat. Übrigens fallen in diese Zeit wichtige Begegnungen. So weilte der bekannte spanische Meteorologe FRANCESCO MORAN-SAMANIEGO in Berlin, machte ERTELS Bekanntschaft, die jahrzehntelang dauerte. Der international bekannte amerikanische Meteorologe JEROME NAMIAS hatte seinerzeit beabsichtigt, in Berlin zu studieren und mit ERTEL zusammenzuarbeiten. In gewisser Weise hatte das frühere Universitäts-Institut vor über 70 Jahren eine sehr nachhaltige internationale Ausstrahlung gehabt, die auch zunehmend mit dem Bekanntheitsgrad von HANS ERTEL zusammenhing, dessen Arbeiten auch in England und USA sehr wohl zur Kenntnis genommen wurden.

Es muss ja auch gesehen werden, dass in der internationalen Wissenschaft Deutsch die Fachsprache war und die „Meteorologische Zeitschrift" eine international führende Zeitschrift gewesen ist. Fast alle international bekannten Meteorologen haben darin publiziert.

Bereits frühzeitig hatte FICKER ERTELS Begabung erkannt, und so versuchte er, ihn an die Meteorologie zu binden. Andererseits hätte auch BARTELS gerne ERTEL an seinem Institut gehabt. So kam es, dass ERTEL kurzfristig in Potsdam arbeitete, jedoch schnell in die Meteorologie zurückkehrte. Aus dieser Zeit stammen auch einige Arbeiten aus dem Interessensgebiet BARTELS, wie z. B. zur Grönland- 
drift, Bewegung von Elektronen in inhomogenen Magnetfeldern sowie zur Polfluchtkraft, Themen, die ERTEL aufgegriffen hat und die auch später in der Literatur immer wieder zitiert wurden.

ERTEL legte FICKER eine Arbeit vor, von der FICKER sagte, er habe sie zwar nicht verstanden; gleichwohl ließ er sie drucken. ERTEL hatte somit mit 25 Jahren seine erste wissenschaftliche Publikation herausgebracht. Nachdem FICKER auf ERTEL aufmerksam geworden war, tat er alles, um diesem den akademischen Weg zu ebnen. In kurzer Zeit absolvierte ERTEL an der Universität in Berlin sein Studium in 6 Semestern und promovierte.

Für die Wirksamkeit ERTELS sollten weitere Umstände günstig sein: FICKER war Sekretär und Mitglied der Berliner Akademie. Hinzu kommt, dass sowohl ALBERT DEFANT (1884-1974) sowie JULIUS BARTELS (1899-1964) der Akademie angehörten. Alle waren bestens bekannt mit MAX VON LAUE (1879-1960), ALBERT EINSTEIN (18791955), MAX PlANCK (1958-1947), ERWIN SCHRÖDINGER (18871961) u. a., denen auch ERTEL vorgestellt wurde. Diese Gelehrten wurden rasch auf ERTEL aufmerksam und förderten ihn nach Kräften. So wundert es nicht, dass ERTEL Themen auch aus dem Umfeld EINSTEINS und SCHRÖDINGERS aufgriff. Dies alles waren aber Nebenschritte, denn ERTEL kehrte zu FICKER zurück und widmete sich ganz der theoretischen Meteorologie.

Aus dem eingangs erwähnten Briefwechsel BJERKNES-FICKER war die Ähnlichkeit, aber auch der Gegensatz beider Persönlichkeiten deutlich geworden. In die nachfolgende Zeit fiel die Diskussion um die Steuerung des Wettergeschehens durch die Stratosphäre, die Frage der Zusammensetzung der Depressionen sowie die weitergehende Ausarbeitung des BJERKNESschen Konzeptes. Anlässlich der 17. Versammlung der Deutschen Meteorologischen Gesellschaft in Wien 1930 hatte sich ERTEL mit diesem Komplex auseinandergesetzt. Kurz danach räumte BJERKNES in seiner „Physikalischen Hydrodynamik" ausdrücklich die Priorität FICKERS bei der Entdeckung der großen Diskontinuitäten für die Wetterentwicklung ein. BJERKNES betonte, dass es die norwegische Schule versäumt hatte, den FICKERschen Ergebnissen rechtzeitig zu folgen. FICKER selbst hatte 1935 nochmals die Frage des Einflusses der Stratosphäre auf die Wetterentwicklung aufgegriffen. In der weiteren Diskussion hat ERTEL insofern eine Rolle gespielt, als er verschiedene theoretische Fragen, wie z. B. zur zonalen Zirkulation, den atmosphärischen Druckschwankungen des Windfeldes an der Tropopause, der Zyklonenbewegung usw., aufgegriffen hat. In zwei Monographien hat ERTEL das theoretische Gebäude der Meteorologie umrissen: „Methoden und 
Probleme der dynamischen Meteorologie“ (1938) und „Die theoretischen Grundlagen der Meteorologie“ (1939). In beiden Bänden würdigt ERTEL den norwegischen Beitrag, aber auch FICKERS Beiträge werden hinreichend dargestellt.

Es wurde bereits erwähnt, dass das Meteorologische Institut in Berlin seit 1936 eine eigene Reihe ,Veröffentlichungen ..." herausgab. Als Herausgeber zeichneten FICKER und ERTEL. Das erste Heft dieser Reihe ist die ERTELsche Abhandlung „Advektivdynamische Theorie der Luftdruckschwankungen und ihrer Periodizitäten“ (1939). Die weiteren Hefte beinhalten u. a. Beiträge von K. BROCKS, H. FiCKER, J. JAW, S. LI, O. SCHNEIDER, K. STUMPFF sowie I. WEISS. Von diesen Mitarbeitern hatte BROCKS später in Hamburg eine leitende Funktion inne, SCHNEIDER war Vorsitzender des argentinischen IGY- und Antarktis-Programms und JAW hatte eine führende Rolle in China inne. Wiederholt wurde ERTEL später auch von der Chinesischen Akademie der Wissenschaften eingeladen, da ihm in China eine ganz besondere Wertschätzung und Verehrung galt.

Im Jahre 1937 übernahm FICKER das Direktorat der Zentralanstalt für Meteorologie und Geodynamik in Wien sowie die Professur für Physik der Erde an der Wiener Universität. In dieser Zeit riss aber die freundschaftliche Verbindung zwischen FICKER und ERTEL nicht ab.

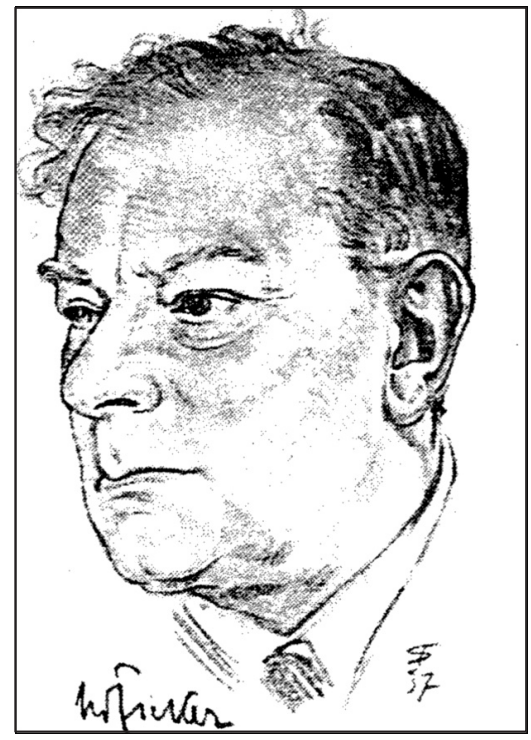

Abb. 1. Heinrich Ficker (Bild im Privatbesitz)

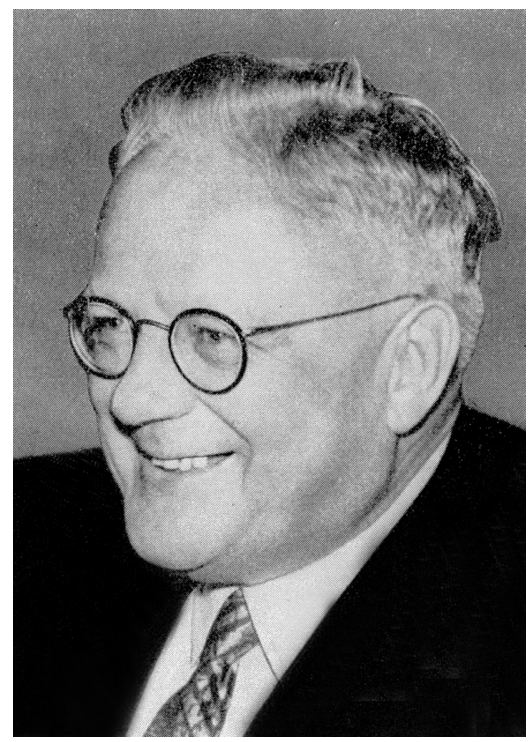

Abb. 2. Hans Ertel (Bild im Privatbesitz) 
ERTEL erhielt eine Planstelle an der Zentralanstalt für Meteorologie in Wien, trat sie aber niemals an, da er sofort für die Meteorologenausbildung in Berlin abgeordnet wurde. Zwischen FICKER und ERTEL bestanden vor allem briefliche Kontakte, jedoch hat FICKER nachdrücklich geholfen, dass ERTEL 1943 Ordinarius an der Universität Innsbruck wurde. Diese Stelle musste er nach 1945 räumen; sie wurde von ALBERT DEFANT eingenommen.

Kurz danach nahm ERTEL am internationalen Projekt der isentropen Analyse am MIT in Cambridge (USA) teil, wobei sich seine Beziehungen zu CARL-GUSTAF ROSSBY wesentlich vertiefen sollten. Die Freundschaft beider Gelehrter hielt ein Leben lang an, und auch später trafen sich beide noch, so u. a. bei der Jubiläumsfeier der Zentralanstalt für Meteorologie in Wien 1950. Später versuchte ROSSBY immer wieder, ERTEL in internationale Forschungsvorhaben der Meteorologie einzubinden. Dass dies nicht gelang, lag sicher daran, dass ERTEL wegen seiner Tätigkeit als Vizepräsident der Deutschen Akademie der Wissenschaften $u$. a. einfach keine Zeit mehr übrig hatte für weitere Verpflichtungen.

Die weitere Zusammenarbeit zwischen ERTEL und FICKER war seit dessen Weggang im Brieflichen zu suchen. Von FICKER wird berichtet, dass er gerne alle schwierigen Probleme sammelte und diese dann an ERTEL schickte. Dann dauerte es, bis er alle erhofften Lösungen erhielt. Zum 60. Geburtstag von H. FICKER veröffentlichte ERTEL 1941 in den „Naturwissenschaften“ einen sehr lesenswerten Beitrag (ERTEL [8]). Zwar bemühte sich FICKER darum, ERTEL nach Wien zu bekommen, doch dieser wollte nicht. ERTEL hatte schon damals alle anderen akademischen Möglichkeiten außerhalb Berlins abgelehnt. Lediglich 1943 nahm ERTEL einen Ruf nach Innsbruck an, den er dann 1945 abgeben musste (s. o.). Auch später hat er Rufe z. B. nach Graz, München und Wien nicht angenommen. Besonders um die Berufung nach Graz sowie Wien hatte sich FICKER bemüht. Er hätte es gerne gesehen, dass z. B. ERTEL sein Nachfolger in Wien werde. Übrigens scheiterte die Berufung nach Graz auf postalischem Wege: Der Brief wurde ERTEL viel zu spät zugestellt und die Stelle anderweitig besetzt. Wenngleich FICKER ERTEL gerne an der Universität Wien gesehen hätte, so wollte dieser Berlin nicht verlassen.

Dessen ungeachtet waren die Beziehungen ERTEL-FICKER in all den Jahren sehr herzlich. Ein Wiedersehen gab es auch anlässlich der 250Jahr-Feier der Deutschen Akademie der Wissenschaften, an der eine Delegation der Österreichischen Akademie der Wissenschaften teilnahm, so u. a. der Generalsekretär KEIL und FICKER. FICKER vertrat 
dabei zugleich auch die Wiener Universität. Stets war FICKER auch daran interessiert, z. B. über ERTELS Beziehung zu ROSSBY zu erfahren. So fragte er ERTEL u. a. in einem Brief vom 29. Januar 1950:

„Es würde mich sehr interessieren, von Dir zu erfahren, wie der Besuch RossBYS außerhalb des Bräustübls ausgefallen ist. Ich kann mich in die neue Meteorologie nicht mehr hineinfinden, wohl auch deshalb, weil noch keine gute Darstellung in deutscher Sprache vorhanden ist. Es berührt mich fast komisch, dass trotz der Ausbildung von REUTER (REUTER war später Professor für theoretische Meteorologie in Wien) in Stockholm und dem Kurs Dr. KLETTERS (Mitarbeiter von FICKER) in Kissingen und ihres Bemühens, in der Synoptik (praktischen Wettervorhersage) nach SCHERHAG und Rossby zu arbeiten, in der Praxis sich doch meine alten Bauernregeln am besten bewähren."

In diesem Zusammenhang mag auch an ein Schreiben von DEFANT an ERTEL vom 17. Juli 1954 erinnert werden, als sich dieser für die Glückwünsche der Deutschen Akademie der Wissenschaften zu seinem 70. Geburtstag bedankt, die ERTEL verfasst hatte. Er schreibt u. a.:

„Ihre Zeilen haben mir die schönen Zeiten meiner erfolgreichsten Jahre in Berlin lebhaft in Erinnerung gebracht und des Zusammenseins mit Ihnen."

Mit der Wahl zum korrespondierenden Mitglied der Österreichischen Akademie der Wissenschaften hatten sich ERTELS Beziehungen zu Österreich weiter vertieft. In dem Wahlvorschlag (1956) lobten FICKER u. a. ERTEL als den besten Theoretiker der Meteorologie im deutschen Sprachraum. ERTEL selbst war über die Wahl sehr erfreut und hatte später bei einer Tagung 1965 nochmals Gelegenheit, Wien wiederzusehen und Freunde der Akademie, u. a. A. DEFANT und F. Steinhauser, zu treffen. FicKers Tod (1957) hat ERTEL sehr betroffen gemacht. In einem ersten Nachruf (ERTEL 1958) schrieb er:

„Mit tiefem Schmerz empfinden wir Meteorologen den Verlust dieses Mannes, in dessen ganzem Wesen die Eigenschaften eines klarblickenden Forschers mit der warmen menschlichen Herzensgüte eines edlen Charakters harmonisch zusammenklangen“" (Zeitschrift für Meteorologie 1958).

An die Österreichische Akademie der Wissenschaften schickte die Deutsche Akademie der Wissenschaften folgenden Brief:

,Die Deutsche Akademie der Wissenschaften zu Berlin bringt in tiefer Trauer der Österreichischen Akademie der Wissenschaften zum Hinscheiden ihres Vizepräsidenten und Präsidenten der mathematisch-naturwissenschaftlichen Klasse Prof. em. Dr. Dr. h.c. HEINRICH FICKER ihr aufrichtiges Beileid zum Ausdruck. Mit dem Dahingeschiedenen hat die meteorologische Wissenschaft einen Forscher von 
höchstem Rang und einen Altmeister ihres Faches verloren, der mit begeistertem Streben nach Erkenntnis der Natur schlichte Vornehmheit seines Charakters, Lauterkeit seiner Gesinnung und tiefinnige Herzensgüte harmonisch verband. Die Deutsche Akademie der Wissenschaften zu Berlin wird diesem hochverdienten Gelehrten und guten Menschen dauernd ein ehrendes Gedenken bewahren. In aufrichtiger Anteilnahme, HANS ERTEL.“

Das waren Worte, die auch für ERTEL selbst galten. Im Jahrbuch der Deutschen Akademie der Wissenschaften für 1962 hat ERTEL dann einen ausführlicheren Nachruf geschrieben (ERTEL [10]).

Fast dreißig Jahre verband FICKER und ERTEL eine besondere, im Laufe der Zeit tief gewachsene Freundschaft, die ihresgleichen sucht. FICKER hat selbstlos den wissenschaftlichen Werdegang ERTELS gefördert. Er hat ihn bekannt gemacht und eingeführt in die wissenschaftliche Welt EINSTEINS, PlanCKS, LAUES, SCHRÖDINGERS u. a., die ihrerseits ERTEL mit Herzlichkeit aufnahmen und umfassend förderten. So konnte sich ERTEL in beispielloser Weise unter der Obhut und Güte weltbekannter Wissenschaftler zu einem hervorragenden Gelehrten entwickeln.

Es mögen noch ein paar Worte angefügt werden von Prof. HEINZ REUTER (Wien), der ein Schüler FICKERS war. Er schrieb mir in einem Brief:

„Nun noch ein paar Worte über FICKER. Er hat mir das Verständnis für die praktische Meteorologie beigebracht. Ich habe ihn immer schon deshalb bewundert, weil er ohne besondere theoretische Kenntnisse die Vorgänge in der Natur beschreiben und klären konnte, was bei der Komplexität der atmosphärischen Prozesse einmalig erscheint. Auch MAX PLANCK soll (so hat mir ERTEL einmal erzählt) gesagt haben, dass er keinen anderen Naturwissenschaftler kennengelernt habe, der fähig gewesen wäre, ohne Hilfe der Theorie Prozessabläufe in der Natur zu beschreiben, und zwar nach einem strengen Kausalprinzip. Wenn ich zurückblicke in die Zeit meiner ersten wissenschaftlichen meteorologischen Arbeiten und die ganze (rasante) Entwicklung unserer Wissenschaft bis heute an meinem geistigen Auge vorbeiziehen lasse, dann glaube ich, behaupten zu können, dass ERTEL die theoretischen Grundlagen für die bis heute so weit entwickelte Theorie geliefert hat und FICKER der wahrscheinlich letzte empirische große Synoptiker war." (REUTER [13].)

\section{Ausblick}

Es ist wiederholt, auch in der Meteorologie, von wissenschaftlichen Schulen die Rede gewesen. Man spricht von der Chicagoer Schule, von der norwegischen Meteorologenschule, so dass gefragt werden kann, wie es im deutschen Sprachraum ist. Sicher hat FICKER, aber 
auch ERTEL, keine wissenschaftliche Schule gebildet. Beide waren sich einig darin, die individuellen Möglichkeiten der ihnen anvertrauten Menschen zu fördern, so gut es ging. Gerade in der Bandbreite der Begabungen sahen sie die Chance für den wissenschaftlichen Werdegang, und so wundert es nicht, dass aus ihrem Zuhörerkreis viele Themen behandelt wurden, die die gesamte Geophysik und Meteorologie abdecken. Gerade darin lag die Ausrichtung dieser beiden akademischen Lehrer. Die Begabungsvielfalt war es, die gefördert wurde.

Später hat sich ERTEL, nach Gründung des Instituts für Physikalische Hydrographie, besonders den praktischen Problemen zugewandt. Das Institut, eine Zeit lang international führend in verschiedenen Teilgebieten der Hydrodynamik und physikalischen Hydrographie, gab außerdem ab 1953 eine eigene Schriftenreihe heraus: die Acta Hydrophysica. Sie sollte die Theoretiker und Praktiker zusammenführen, wie dies schon LEIBNIZ gefordert hatte. Im Laufe der Jahre ERTEL gab die Zeitschrift bis 1971 heraus - erschienen auch viele Arbeiten, die sowohl den theoretischen Aspekt als auch den praktischen Nutzen für die Volkswirtschaft berücksichtigten. Überdies wurde die Zeitschrift als Tauschobjekt mit anderen Institutionen in aller Welt genutzt, wodurch ERTELS Institut sehr viele ausländische Bücher und Zeitschriften erhielt, etwas, was damals durchaus sehr wichtig war. Interessant ist auch, dass, fast an FICKERS praktische Richtung erinnernd, auch ERTEL die Forschungsthematik des Instituts praxisorientiert sah. Lange bevor überhaupt in der Geophysik von ökologischen Fragestellungen gesprochen wurde, hatte das Institut für Physikalische Hydrographie in Berlin international bedeutende Probleme der Geo-Ökologie sowie des Umweltschutzes in den Grundzügen bearbeitet. Hierzu zählt auch die vom Institut herausgegebene „Quellensammlung zur Hydrographie und Meteorologie", die CURT WEIKINN, der von ERTEL ans Institut geholt worden war, erfolgreich zusammenstellen konnte. Das Werk hat höchste internationale Anerkennung gefunden, so z. B. in Rezensionen von H. E. LANDSBERG (USA), H. LAMB (England) und A. RETHLY (Ungarn) u. a.

Für die Entwicklung der Meteorologie war die Begegnung von FICKER mit ERTEL ein Glücksfall. Dadurch, dass FICKER ERTEL sozusagen ,entdeckte“, dessen Werdegang positiv begleitete und ERTEL sich somit umfassend entwickeln konnte, wuchs der Erkenntniszuwachs in den meteorologischen und geophysikalischen Disziplinen. FICKERS und ERTELS Beziehung ist ein Beispiel der harmonischen Entwicklung eines gütigen Lehrers für seinen 
Schüler, wobei sich dieser ganz im Geiste FICKERS ebenso gegenüber allen verhielt, die sich auf den Weg der Wissenschaft machten.

\section{Ungedruckte Quellen}

Die Briefe FICKER/BJERKNES befanden sich vor Jahren in der Handschriftenabteilung der UB Oslo. Die Briefe ERTEL/FICKER sind im Privatbesitz. ERTELS Brief sowie der Wahlvorschlag an die ÖAW befinden sich in deren Archiv (Personalakt HEINRICH VON FICKER).

\section{Danksagung}

Der Universitätsbibliothek Oslo sowie dem Archiv der Österreichischen Akademie der Wissenschaften danke ich für freundliche Hilfe. Herrn Professor HELMUT MORITZ (Graz) danke ich, dass er mir nachdrücklich bei Verbesserungen half, für die er sich wieder Rat bei Professor HeINZ KAUTZLEBEN (Berlin) holte.

\section{Bemerkung}

Eine Vorveröffentlichung erschien im Internet unter http://verplant.org/historygeophysics/Ficker.htm. Die Leibniz-Sozietät der Wissenschaften in Berlin hat in gleicher Weise des hervorragenden Meteorologen HEINRICH FICKER gedacht. Eine andere Fassung dieses Beitrages wurde der Leibniz-Sozietät vorgelegt.

\section{Literatur}

[1] BJerknes, V., BJerknes, J., Solberg, H., Bergeron, T. (1933) Physikalische Hydrodynamik mit Anwendung auf die dynamische Meteorologie. Springer, Berlin

[2] ERTEL, H. (1932) Theorie der durch Variationen des magnetischen Potentials induzierten Erdströme bei ungleichförmiger Leitfähigkeit der Erdrinde (Inaugurationsdiss. 1932). Archiv f. Erdmagnetismus Heft 8

[3] ERTEL, H. (1931) Der Einfluß der Stratosphäre auf die Dynamik des Wetters. Meteorol. Z. 48: 461

[4] ERTEL, H. (1936) Singuläre Advektion und ihre Darstellung durch C. G. Rossbys Advektionsfunktion. Veröff. Meteorol. Inst. U. Berlin I, Heft 6

[5] ERTEL, H. (1938) Methoden und Probleme der dynamischen Meteorologie. Springer, Berlin

[6] ERTEL, H. (1939) Die theoretischen Grundlagen der dynamischen Meteorologie. Meteorol. Taschenbuch, V. Ausg., herausg. von F. LINKE. Bahrt, Leipzig

[7] ERTEL, H. (1940) Elemente der Operatorenrechnung mit geophysikalischen Anwendungen. Springer, Berlin

[8] ERTEL, H. (1941) Heinrich von Ficker zu seinem 60. Geburtstag am 22. November 1941. Naturwiss. 29: 697

[9] ERTEL, H. (1957) Schreiben an die Österreichische Akademie der Wissenschaften (Archiv Österr. Akad. Wiss., Personalakt Heinrich von Ficker, Mappe 1) 
[10] ERTEL, H. (1963) Heinrich Ficker. Jahrbuch der Deutschen Akad. Wiss. zu Berlin, 1962. Akademie-Verlag, Berlin

[11] FICKER, H. (1935) Der Einfluß der Stratosphäre auf die Wetterentwicklung. Naturwiss. 23: 552

[12] Planck, M. (1948) Max Planck in seinen Akademie-Ansprachen. Erinnerungsschrift der Deutschen Akademie der Wissenschaften zu Berlin. AkademieVerlag, Berlin

[13] ReuTER, H. (1987) Brief an den Verfasser vom 12. 12. 1987

[14] SCHRÖDER, W. (1971) Nachruf auf Hans Ertel. Wetter und Leben, Heft 4

Anschrift des Verfassers: Dr. Wilfried Schröder, Geophysikalische Station, Hechelstraße 8, 28777 Bremen, Deutschland. 